\title{
Mobile Vehicular Edge Computing Architecture using Rideshare Taxis as a Mobile Edge Server
}

\author{
Mohammed Laroui* ${ }^{\ddagger}$, Boubakr Nour ${ }^{\llbracket}$, Hassine Moungla ${ }^{\ddagger \S}$, Hossam Afifi ${ }^{\S}$, and Moussa Ali Cherif* \\ *EEDIS Laboratory, Computer Science Departement, Djillali Liabes University, Sidi Bel Abbes, Algeria \\ $\ddagger$ Université de Paris, LIPADE, F-75006 Paris, France \\ $\S$ UMR 5157, CNRS, Institut Polytechnique de Paris, Telecom SudParis Saclay, France \\ ISchool of Computer Science, Beijing Institute of Technology, Beijing, China \\ Emails: \{mohammed.laroui,moussa.alicherif\}@univ-sba.dz, \\ $\{$ mohammed.laroui, hassine.moungla\}@ parisdescartes.fr, \\ n.boubakr@bit.edu.cn, hossam.afifi@ telecom-sudparis.eu
}

\begin{abstract}
We propose to utilize rideshare taxis as infrastructure for both communication and computation. Rideshare overlays become hence Mobile Edge Nodes. End-users utilize near rideshare taxis as edge servers to receive video chunks for live video streaming. The set cover problem (SCP) is used to formulate the rideshare taxis coverage optimization inside the city. It provides the maximum number of rideshare taxis that cover end-users routes which guarantee the efficiency of communication services. Simulation results show that the proposed architecture dramatically enhances the quality of service and the overall communication performance in terms of execution time and energy consumption.
\end{abstract}

Index Terms-Vehicular Edge Computing, Rideshare Taxis, Energy Management.

\section{INTRODUCTION}

We propose an extended 'edge computing' paradigm on top of a typical rideshare vehicular network. We design a fully distributed mobile vehicular edge architecture that takes rideshare taxis as infrastructure. This architecture has many of features that make it feasible and more scalable in real deployment including the geo-distribution [1], [2], low-latency communication [3], ubiquitous data caching and processing [4], [5]. The rideshare taxis coverage problem is formulated as a lineaire programming model where Set Cover Problem (SCP) [6] is used to maximize the coverage in order to maintain the service. We use a route prediction algorithm developed in [7], it predicts probable future positions (road crossings) for vehicles in city of Rome. In addition the cloud energy consumption (CEC) is measured with and without edge utilization in two different cases. Beside, the set cover problem is compared with Maximum Coverage Problem (MCP) [8] in terms of execution time.

\section{MVEC: Mobile Vehicular Edge Computing ARCHITECTURE}

This section describes the proposed Mobile Vehicular Edge Computing (MVEC) architecture. Figure 1 illustrates a generic view of MVEC. In addition to cloud servers responsible for original data recovery and synchronization; we propose the

Mr. Laroui is corresponding author. use of rideshare taxis as mobile infrastructure servers (mobile edge), tending to distribute the video chunks and providing a ubiquitous content distribution.

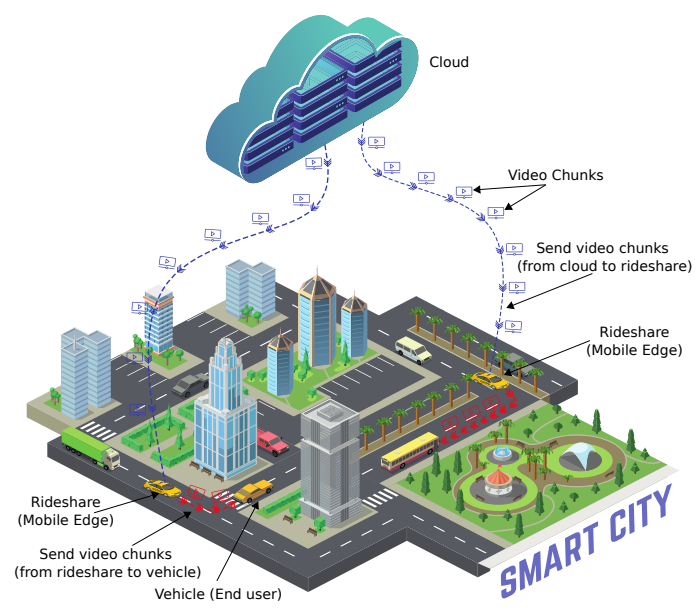

Fig. 1: MVEC architecture overview.

\section{iII. Problem Formulation \& Proposed Solution}

The objective in this work is to maximize the shared path distance between the vehicle and the rideshare taxis with the minimum number of rideshare taxis to guarantee the efficiency of the service. Let $S=\left\{s_{1}, s_{2}, s_{3}, \ldots, s_{n}\right\}$ a family of sets represent the rideshare taxis routes, $E=\left\{e_{1}, e_{2}, e_{3}, \ldots, e_{m}\right\}$ the representation of the vehicle route as a set of segments $e$ inside the city where $\bigcup S=E$, and $H=\left\{h_{1}, h_{2}, h_{3}, \ldots, h_{4}\right\}$ a set of handovers correspond to rideshare taxis route. The objective is to find a subset $X$ of $S$ such that $\bigcup X=E$ and $|X|, h(\bigcup X)$ are small as possible. The formulation of the problem based on SCP can be expressed as follow:

$$
\min \sum_{i=1}^{n} x_{i} h_{i}
$$

Subject to:

$$
\sum_{\left\{i \mid e_{j} \in s_{i}\right\}} x_{i} \geq 1 \quad \forall e_{j} \in E
$$




$$
x_{i} \in\{0,1\}, \forall i \in\{1 \ldots n\}
$$

- Complexity: The formulated problem is an adaptation of the SCP, which is an NP-complete. For this, the algorithm 1 use only a subset from the global set of rideshare taxis paths, this subset contain only the rideshare taxis that shared parts of the path with the vehicle which can reduce the complexity in terms of data processing, consequently reduce the execution time.

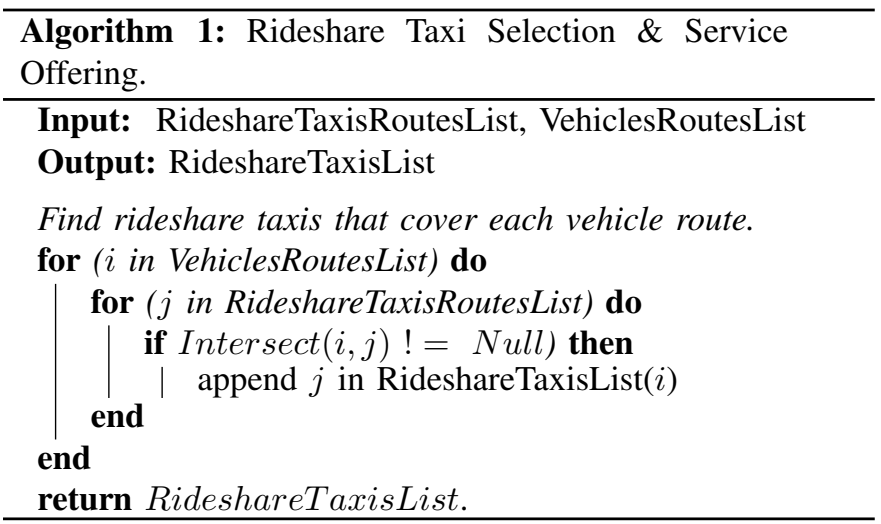

\section{PERformance Evaluation}

The performance of MVEC architecture has been evaluated using different scenarios in a simulated environment. We have used Cloud Report ${ }^{1}$ to simulate both rideshare and enduser communications with the cloud, and $\mathrm{SUMO}^{2}$ for urban mobility, as well as real tracking data of Rome city.

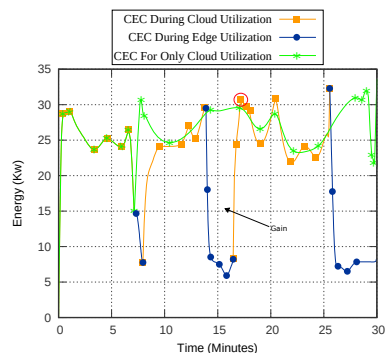

(a) Route 1

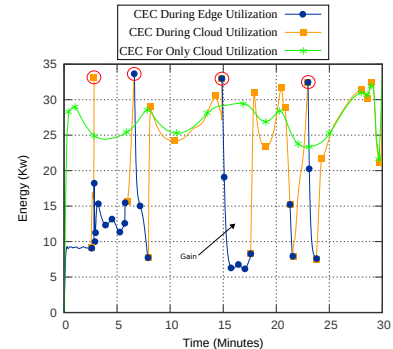

(b) Route 2
Fig. 2: CEC with/out Edge Servers.

Figure 2 displays the cloud energy consumption (CEC) with and without edge servers for two different routes. In Figure $2 \mathrm{a}$ we show that the energy consumption during cloud utilization and only cloud utilization is the same in the first 14 minutes because no coverage exists, after that, we can see that the energy is decreased when the edge server in the rideshare taxis is used and hence increases energy gain.

Similarly, in Figure 2b we show that the rideshare with high coverage leads to a decrease of cloud energy consumption and

\footnotetext{
${ }^{1}$ Cloud Report: www.github.com/thiagotts/CloudReports

${ }^{2}$ SUMO: www.sumo.dlr.de/docs/index.html
}

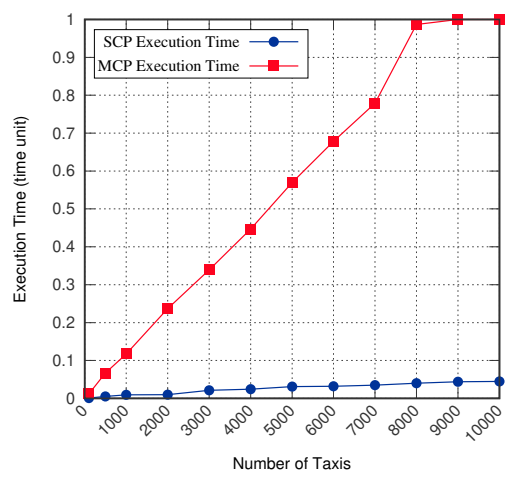

Fig. 3: MCP \& SCP Execution Time.

at the same time increase the gain of energy. Also we show that when no coverage exists, the energy consumption of cloud increase dramatically (red circles in Figures $2 \mathrm{a}$ and $2 \mathrm{~b}$ ) caused by the handover during the communication which leads to send a large number of requests to the cloud.

Figure 3 displays the execution time of the maximum coverage problem (MCP) and the set cover problem (SCP), we show that the execution time of the two algorithms is high when the number of rideshare taxi is increase, but the execution time of SCP is less than the MCP.

\section{CONClusion \& Future Work}

In this poster, we have presented a distributed Mobile Vehicular Edge Computing (MVEC) architecture taking the benefits of rideshare taxis as infrastructure. With the increase of ride-sharing vehicles and their distribution in different cities, the proposed architecture can scale to cover big cities. The proposed architecture is promising to reshape the vehicular environment, and enhance both the communication and quality of service in large-scale scenarios. The next stage of our research will be deployment of the proposed architecture in a real scenario, and extending mobile rideshare taxis to buses and other public transportation networks. Thus, the selection will be based on advanced multi agent reinforcement learning algorithms that take into consideration traffic congestion, users and taxis itinerary.

\section{REFERENCES}

[1] R. Zhang et al., "A reliable and energy-efficient leader election algorithm for wireless body area networks," in IEEE ICC, 2015, pp. 530-535.

[2] H. Moungla et al., "Cost-effective reliability-and energy-based intraWBAN interference mitigation," in IEEE GLOBECOM, 2014.

[3] H. Moungla et al., "Distributed interference management in medical wireless sensor networks," in IEEE CCNC, 2016, pp. 151-155.

[4] H. Khelifi et al., "Named data networking in vehicular ad hoc networks: State-of-the-art and challenges," IEEE Commun. Surveys Tuts., 2019.

[5] M. Laroui et al., "Driving path stability in VANETs," in IEEE GLOBECOM, 2018, pp. 1-6.

[6] R. M. Karp, "Reducibility among combinatorial problems," in Complexity of computer computations. Springer, 1972, pp. 85-103.

[7] M. Laroui et al., "Energy Management For Electric Vehicles in Smart Cities: A Deep Learning Approach," in IEEE IWCMC, 2019, pp. 1-6.

[8] S. Khuller et al., "The budgeted maximum coverage problem," Inf. Process. Lett., vol. 70, no. 1, pp. 39-45, 1999. 